\title{
Seasonality of Hair Shedding in Healthy Women Complaining of Hair Loss
}

\author{
Michael Kunz $^{\mathrm{a}}$ Burkhardt Seifert ${ }^{\mathrm{b}}$ Ralph M. Trüeb ${ }^{\mathrm{a}}$ \\ ${ }^{a}$ Department of Dermatology, University Hospital of Zürich, and ${ }^{b}$ Biostatistics Unit, Institute for Social and \\ Preventive Medicine, University of Zürich, Zürich, Switzerland
}

\section{Key Words}

Hair shedding, seasonality $\cdot$ Hair loss, women

\begin{abstract}
Background: A number of otherwise healthy women with or without clinical alopecia complain of recurrent hair loss, presumably reflecting seasonality in the growth and shedding of hair. Objective: To test the hypothesis that periodicity in hair shedding reflects seasonal changes in human hair growth. Methods: Retrospective case study over a period of 6 years of apparently healthy women with the complaint of hair loss. All underwent biochemical investigations, and trichograms were made. Results: After exclusion of patients with a disease or on drugs known to cause hair loss, 823 women remained. Analysis of trichograms demonstrated annual periodicity in the growth and shedding of hair, manifested by a maximal proportion of telogen hairs in summer. A second peak seems to exist, though it is less pronounced, in spring. The telogen rates were lowest in late winter. Conclusions: These results confirm the findings of former authors who have indicated seasonal changes in human hair growth, though this is the first study performed systematically in a representative number of women.
\end{abstract}

Copyright $\odot 2009$ S. Karger AG, Basel
(C) 2009 S. Karger AG, Basel

$1018-8665 / 09 / 2192-0105 \$ 26.00 / 0$

Fax +4161306 1234

E-Mail karger@karger.ch

www.karger.com
Accessible online at: www.karger.com/drm

\section{Introduction}

Few dermatological complaints carry as much emotional overtones as hair loss. Frequently encountered in dermatological practice is the woman who is 'losing her hair'. Many factors can lead to pathologically increased hair loss. A definitive diagnosis can usually be established, particularly focussing on the chronology of preceding events, examination of the scalp, pattern of hair loss, results of the pull test, examination of the bulbs of the shed hairs and a few pertinent screening blood tests [1]. In most patients presenting in clinical practice, hair loss results from an increase in the percentage of follicles in the telogen phase with subsequent increased shedding of the associated club hairs (telogen effluvium). Once the diagnosis is established, treatment appropriate for that diagnosis is likely to control the hair loss, e.g. iron deficiency, other dietary deficiencies, thyroid disease, other metabolic or endocrine diseases, systemic lupus erythematosus, other connective tissue disorders, syphilis, other chronic infectious diseases, and drug-induced telogen effluvium.

In otherwise healthy women, female-pattern hair loss (FPHL) is the most frequent cause of telogen effluvium and is usually successfully treated with topical minoxidil

Prof. Ralph M. Trüeb, MD

Department of Dermatology, University Hospital of Zürich

Gloriastrasse 31

CH-8091 Zürich (Switzerland)

Tel. +41 44255 3079, Fax +41 44255 4431, E-Mail ralph.trueeb@usz.ch 


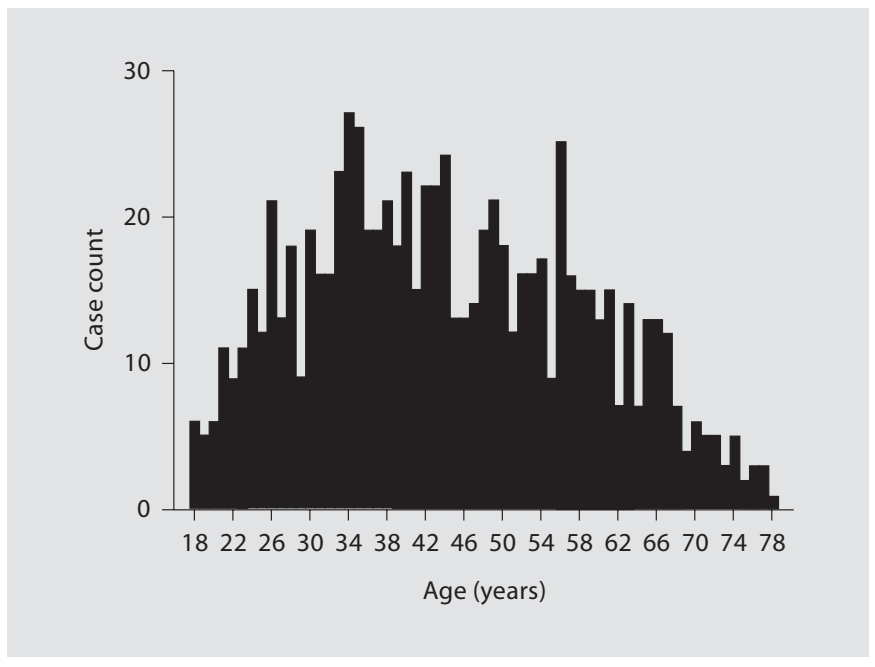

Fig. 1. Distribution of case counts by age.

[2-4]. Special attention is also given to the use of a contraceptive pill or hormonal replacement therapy, since gestagens with proandrogenic effect may exacerbate hair loss and are consequently contraindicated in these women. Some authors eventually recommend the use of antiandrogen therapy, such as cyproterone acetate [5], spironolactone [5, 6] or finasteride [7], though there exists controversy on this issue [8-10]. Nevertheless, a number of women complain of recurrent hair loss, despite at times successful therapy. The condition tends to run a fluctuating course, presumably reflecting seasonal periodicity in the growth and shedding of hair.

To test the hypothesis that periodicity in shedding of hairs in otherwise healthy women complaining of hair loss reflects seasonal changes in human hair growth, we performed a study of telogen rates in relation to the season as assessed by the trichogram technique.

\section{Patients and Methods}

Telogen rates as assessed by the trichogram technique for evaluation of hair loss in women presenting at the Department of Dermatology, University Hospital of Zurich hair clinic, were collected between September 1, 2001, and August 31, 2007. For the trichogram, all patients had at least 50 hairs plucked from sampling sites on the frontal and occipital scalp, using a tightly closing epilation forceps. Uniformly, patients were instructed to leave their hair unwashed and untreated with any cosmetics for 5 days prior to the plucking of the hairs for the trichogram. Immediately after epilation, the hair roots were embedded in Histokitt ${ }^{\mathrm{TM}}$ mounting medium which had previously been spread onto a glass slide for light microscopy. The hair roots were arranged neatly in juxtaposition and covered with a coverslip. Quantification of the different hair roots on the basis of their morphological characteristics relating to the hair cycle was performed by the same investigator.

In all women, a careful history of systemic disease or drugs known to cause hair loss was obtained. Biochemical investigations included thyroid function tests, C-reactive protein and serum ferritin levels in all patients, renal and liver function tests, hormonal and extended nutritional studies in selected cases, as indicated by the patient history, clinical and trichogram findings. Serum levels of ferritin $>40 \mu \mathrm{g} / \mathrm{l}$, vitamin $\mathrm{B}_{12} 300-1,000 \mathrm{ng} / \mathrm{l}$, folic acid 5-40 nM, estradiol (day 21) $>300 \mathrm{pM}$ and progesterone (day 21) $>30 \mathrm{nM}$ were considered normal [11].

Trichograms were included irrespective of the presence or not of clinically appreciable alopecia. Patients with alopecia areata or scarring alopecia were excluded. FPHL was diagnosed either clinically (Ludwig patterns I-III with or without pathological trichogram) or on the basis of a trichogram with a frontal telogen rate $>15 \%$ and an occipital telogen rate $<15 \%$, diffuse telogen effluvium was diagnosed when the occipital telogen rate was above $15 \%$. Patients with dystrophic effluvium (>10\% dystrophic anagen hairs) were excluded. The trichograms were either made on the occasion of the initial visit, without treatment, or at least 6 months after introduction of topical $2 \%$ minoxidil therapy in women with FPHL complaining of hair loss (control trichogram).

\section{Statistical Analysis}

Telogen rates were analyzed with respect to seasonal fluctuations in relation to the period of the year. We also wanted to find out whether telogen rates in relation to the period of the year depended on patient age, and whether there were differences between the single years from 2001 to 2007.

The function GAM (generalized additive model) in the package MGCV of the statistical program R (http://www.r-project. org/) was used for statistical analyses and plots. MGCV means 'GAMs with GCV smoothness estimation and GAMMs by REML/PQL' and GAM means 'generalized additive models with integrated smoothness estimation'. Smooth terms are represented using penalized regression splines with smoothing parameters selected by GCV/UBRE. Wald tests were performed for the analysis of parametric and smooth terms. $\mathrm{p}<0.05$ was considered statistically significant.

\section{Results}

After exclusion of patients with a history of systemic disease or on drugs known to cause hair loss, with abnormal laboratory investigations, alopecia areata or scarring alopecia, 823 otherwise healthy women remained. The mean age of the total group of 823 women was 44.3 years (range: 18-78; fig. 1).

The mean frontal telogen rate was $18.1 \%$ (range: $1-70$ ), and the mean occipital telogen rate was $18.1 \%$ (range: $1-$ 74). The number of women with FPHL was 652 (79.2\%), 


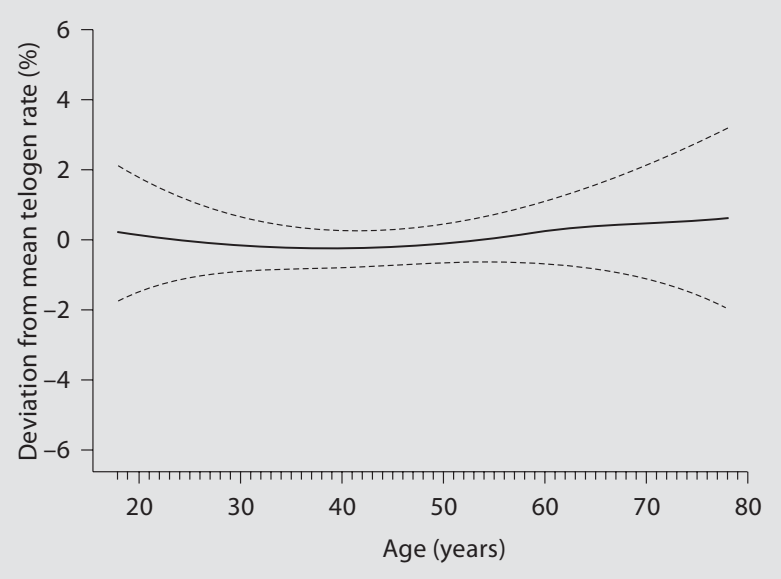

Fig. 2. No fluctuation in frontal telogen rate in relation to patient age.

with a mean age of 44.5 years (range: $18-78$ ). In this group, the mean frontal telogen rate was $18.1 \%$ (range: 1-53). The mean occipital telogen rate in patients with FPHL was $17.6 \%$ (range: $2-73$ ), reflecting a frequent combination of FPHL with telogen effluvium. Telogen rates did not significantly depend on patient age (fig. 2) and year (data not shown).

Telogen rates by month of the year showed an overall annual periodicity, manifested by a maximal proportion of telogen hair in July. A second peak seems to exist, although less pronounced, in April (fig. 3). Fluctuations in telogen rates in relation to the day of the year were found to be highly significant ( $p<0.001$; fig. 4$)$. This was reflected in subsequent clinical images taken of patients (fig. 5). When frontal telogen rates in relation to the period of the year of women with FPHL were separately analyzed, the fluctuations in telogen rates were practically identical to the total group of women.

\section{Discussion}

The hair follicle is subject to constant turnover in the course of perpetual cycles through phases of proliferation (anagen), involution (catagen) and resting (telogen), with regeneration in the successive hair cycle. In the telogen phase, the hair shaft matures into a club hair, which is held tightly in the bulbous base of the follicular epithelium, before it is eventually shed (teloptosis) [12, 13]. There are considerable variations in length of these phases de-

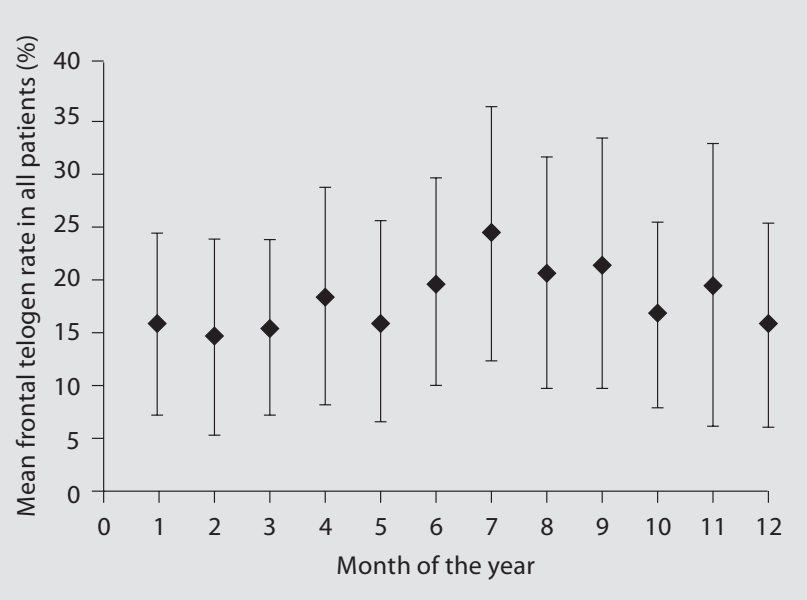

Fig. 3. Means of frontal telogen rates in percent, with their standard deviations, by month of the year $(n=823)$.

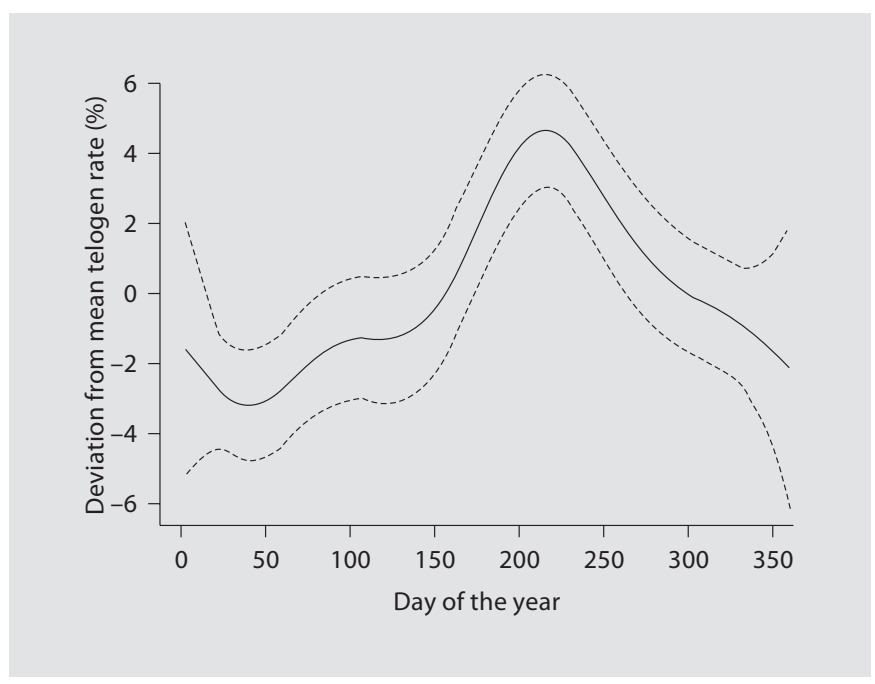

Fig. 4. Fluctuations in frontal telogen rates $(n=823)$ in relation to the day of the year.

pending on the body site location, with the duration of the anagen phase determining the length of the produced hair fiber and the percentage of growing hairs. On the scalp, hairs remain in the anagen phase for a 2- to 6-year period of time, whereas that of telogen is approximately 100 days, resulting in a ratio of anagen to telogen hairs of 9:1. Cyclic hair growth activity occurs in a random mosaic pattern so that on average, the amount of new scalp 

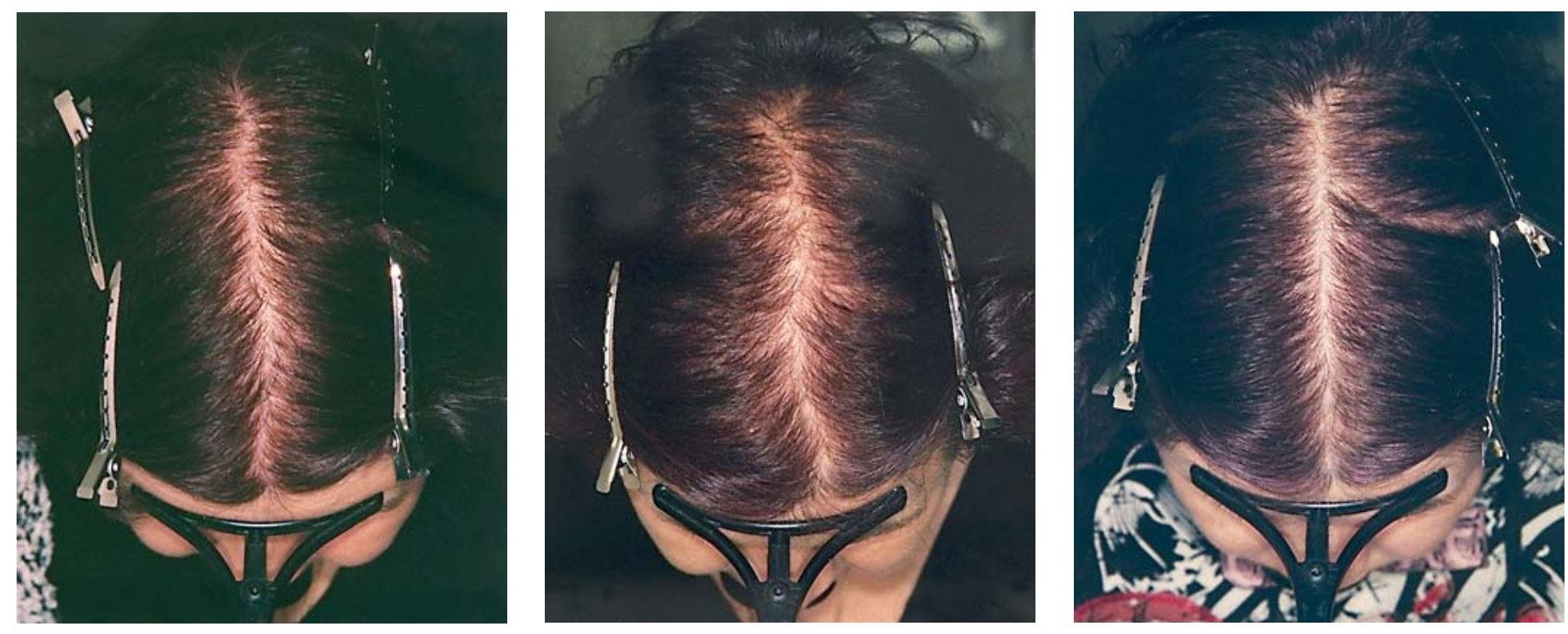

Fig. 5. Subsequent images taken in January 2007, August 2007 and February 2008.

hair formation matches the amount that is shed, thereby maintaining a consistent covering.

Each follicle possesses its own individual control mechanism over the evolution and triggering of the successive phases, though systemic factors, such as the hormonal system, cytokines and growth factors, as well as external factors linked to the environment, toxins, deficiencies of nutrients, vitamins and energy, have influence. In general, the pathological dynamics of hair loss can be related to disorders of hair cycling [14]. Whatever the cause, the follicle tends to behave in a similar way, with telogen effluvium representing the most frequent cause of hair loss [15]. Headington [16] proposed a classification of telogen effluvium into different functional types based on changes in the different phases of the hair cycle. Basically, telogen effluvium results from synchronization phenomena of the hair cycle resulting in increased shedding of hairs from the telogen phase of the cycle, or from shortening of the duration of the anagen phase (without synchronization) underlying androgenetic alopecia.

Headington [16] proposed that a delayed telogen release underlies moulting in mammals, and possibly mild telogen effluvia following travel from low-daylight to high-daylight conditions. In this case, hair follicles remain in a prolonged telogen phase rather than being shed and recycling into anagen. When finally teloptosis sets in, again the clinical sign of increased shedding of club hairs is observed.
Different methods have been adapted for identification and quantification of hair loss. Of these, the trichogram is the first technique standardized for this purpose and is routinely used at our institution for diagnostic purposes in women complaining of hair loss. Initially developed in 1975 by van Scott's group, subsequently other authors modified and standardized the technique defining uniform and strict criteria for reliably assessing the different morphological hair root structures to ensure comparable results $[17,18]$. Telogen rates of $10-15 \%$ (max. $20 \%$ ) are considered normal in the adult scalp.

Our study of 823 otherwise healthy women with telogen effluvium presenting between September 1, 2001, and August 31, 2007 (6 years), in the Hair Consultation Clinic of the University Hospital of Zurich Department of Dermatology demonstrated the existence of overall annual periodicity in the growth and shedding of hair, manifested by a maximal proportion of telogen hairs in July. Taking a telogen phase duration of approximately 100 days into account, one would expect shedding of these hairs by autumn. A second peak seems to exist, although less pronounced, in April. The telogen rate was lowest towards the beginning of February. These results confirm the findings of authors who have formerly demonstrated seasonal changes in human hair growth, though this is the first study performed systematically in women: Orentreich [19] reported 3 women in New York who experienced maximum hair loss in November. By studying a group of 14 men during 18 months, Randall and Ebling 
[20] showed that the proportions of telogen hair and of hair shedding were maximal in September. Courtois et al. [21] observed 10 men, with or without alopecia, for a period of between 8 and 14 years, and also demonstrated a maximal proportion of telogen hairs at the end of summer. Some subjects also exhibited a periodicity approximately corresponding to two annual peaks. Our observation of a far larger number of patients led to statistically more significant calculations of these variations. Since we did not perform the study in a prospective manner on a group of women who were followed for a certain period, but in women visiting our Department with the complaint of hair loss over a period of 6 years, the finding of statistically significant signals of annual periodicity points to a more general phenomenon, irrespective of the presence of clinical FPHL or not, and irrespective of topical minoxidil treatment. Accordingly, fluctuations of telogen rates in relation to the period of the year were practically identical in women with FPHL and in the total group of women. It is our experience that women with FPHL tend to complain more often of noticeable periodicity of hair loss. Due to a reduction of the anagen phase, mathematically, synchronization phenomena can be expected to be more marked in women with FPHL. In these women, the seasonal hair loss may be as striking as to become clinically apparent (fig. 5). The wide range of telogen rates from as low as $1 \%$ to as high as $>70 \%$ is explained by extreme synchronization phenomena in the anagen or telogen phase, respectively, since systemic diseases and alopecia areta were excluded.

The cyclical activities of the hair follicle constitute the mechanism by which mammals change their coat of hair to meet the exigencies of growth, seasonal changes in the ambient environment and, perhaps, normal wear and tear. It seems likely that environmental factors, such as the photoperiod, are mediated through the optic pathway and the neuroendocrine system coat phenotype and function to photoperiod-dependent environmental changes. The fact that human hair follicles, just as those of other mammals, undergo cyclical activity and are influenced by hormones implies that human hair is not unaffected by these phenomena. From an evolutionary point of view, the maintenance of the low winter level of hair shedding and the postponement of hair fall until the end of summer might, perhaps, be postulated as having a selective advantage with respect to insulation of the head against the cold in winter, and protection of the scalp against the midday sun in summer.

More importantly, the existence of seasonal fluctuations in hair growth and shedding complicates the assessment of pharmacological effects. Awareness of these fluctuations is prerequisite to providing the correct cause and prognosis to the patient, ensuring patient compliance with therapy, but it also has potentially serious implications for investigations with new hair-growth-promoting agents: depending on the stage of periodicity in growth and shedding of hair for a particular subject, the heterogeneity of included subjects may be enough to distort the clinical efficacy results and the perceived benefit of an investigational agent. In the active stage of seasonal telogen effluvium, the involved hair follicles would probably fail to respond to the therapeutic agent, which may cause a false-negative result. In the recovery stage, the increased amounts of spontaneously regrowing hair might be interpreted falsely as a positive result.

In summary, this is the first study on seasonal changes in hair growth performed in a representative number of otherwise healthy women with or without clinical FPHL complaining of hair loss, demonstrating a significant seasonal periodicity in the growth and shedding of hair.

\section{References}

-1 Sinclair R: Diffuse hair loss. Int J Dermatol 1999;38(suppl 1):8-18.

-2 De Villez RL, Jacobs JP, Szpunar CA, Warner ML: Androgenetic alopecia in the female: treatment with $2 \%$ topical minoxidil solution. Arch Dermatol 1994;3:303-307.

-3 Lucky AW, Piacquadio DJ, Ditre CM, et al: A randomized, placebo-controlled trial of 5\% and $2 \%$ topical minoxidil solutions in the treatment of female pattern hair loss. J Am Acad Dermatol 2004;50:541-553.
4 Price VH, Menefee E: Quantitative estimation of hair growth. I. Androgenetic alopecia in women: effect of minoxidil. J Invest Dermatol 1990;95:683-687.

-5 Sinclair R, Wewerinke M, Jolley C: Treatment of female pattern hair loss with oral antiandrogens. Br J Dermatol 2005;152:466473.

6 6 Hoedemaker C, van Egmond S, Sinclair R: Treatment of female pattern hair loss with a combination of spironolactone and minoxidil. Australas J Dermatol 2007;48:43-45.
Iorizzo M, Vincenzi C, Voudouris S, Piraccini BM, Tosti A: Finasteride treatment of female pattern hair loss. Arch Dermatol 2006; 142:298-302

-8 Price VH, Roberts JL, Hordinsky M, et al: Lack of efficacy of finasteride in postmenopausal women with androgenetic alopecia. J Am Acad Dermatol 2000;43:768-776. 
\$ Whiting DA, Waldstreicher J, Sanchez M, Kaufman KD: Measuring reversal of hair miniaturization in androgenetic alopecia by follicular counts in horizontal sections of serial scalp biopsies: results of finasteride $1 \mathrm{mg}$ treatment of men and postmenopausal women. J Invest Dermatol Symp Proc 1999;4: 282-284.

10 Vexiau P, Chaspoux C, Boudou P, et al: Effects of minoxidil $2 \%$ vs cyproterone acetate treatment on female androgenetic alopecia: a controlled, 12-month randomized trial. $\mathrm{Br}$ J Dermatol 2002;146:992-999.

11 Rushton DH: Investigating and managing hair loss in apparently healthy women. Can J Dermatol 1993;5:455-461.
2 Paus R, Cotsarelis G: The biology of hair follicles. N Engl J Med 1999;341:491-497.

13 Piérard-Franchimont C, Piérard GE: Teloptosis, a turning point in hair shedding biorhythms. Dermatology 2001;203:115-117.

14 Paus R: Control of the hair cycle and hair diseases as cycling disorders. Curr Opin Dermatol 1996;3:248-258.

15 Kligman AM: Pathologic dynamics of human hair loss. I. Telogen effluvium. Arch Dermatol 1961;83:175-198.

16 Headington JT: Telogen effluvium: new concepts and review. Arch Dermatol 1993;129: 356-363.

17 Braun-Falco O, Heilgemeier CP: The trichogram: structural and functional basis, performance, and interpretation. Sem Dermatol $1985 ; 4: 40-52$.
18 Blume-Peytavi U, Orfanos CE: Microscopy of the hair - the trichogram; in Drup J, Jemec GBE (eds): Handbook of Non-Invasive Methods and the Skin. London, CRC Press, 1995, pp 549-554.

19 Orentreich N: Scalp replacement in man; in Montagna W, Bobson RL (eds): Advances in Biology of Skin. Oxford, Pergamon, 1969, vol IX: Hair Growth, pp 99-108.

20 Randall VA, Ebling FJG: Seasonal changes in human hair growth. Br J Dermatol 1991;124: 146-151.

21 Courtois M, Loussouarn G, Hourseau S, Grollier JF: Periodicity in the growth and shedding of hair. Br J Dermatol 1996;134: 47-54. 